\title{
Design and Implementation of Experimental Platform for Multiple Games
}

\author{
Han Xiao ${ }^{1, a^{*}}$, Wang $Y u^{1, b}$ and Zhang $Y u^{1, c}$ \\ ${ }^{1}$ School of Information Engineering, Yangzhou University, Jiangsu, China \\ a949401659@qq.com, byzdxwangyu@163.com, ${ }^{\mathrm{c}} 1097158540 @ q q . c o m$
}

\section{Keywords: Multiple games Experimental Platform ASP.NET}

Abstract. In recent years, with the in-depth study of the Experimental Economics, an increasing number of game researches need to be done through the computer for online interactive and completing a series of operations such as data record, calculation and feedback. But a lot of experimental researches only under one game structure, in order to more realistic situation, the subjective game learning process under the situation of multiple games need to study, thus the multiple games experimental platform design and implementation. The administrator can set the matching method of the data: random or fix matching. In addition, administrators can also set the number of game structure to achieve true multiple games. The platform based on ASP.NET is b/s structure, it can use IIS release. Within the same local area network, participants only need enter the platform Web site to participate in the experiment, which solve the game experiment space constraints, improve the efficiency of the game experiment, simulate realistic situation completely, and realize experimental study on multiple games.

\section{Introduction}

With the in-depth study on subjective game at home and abroad, the researchers usually analyze the game theory by observing the participants behavior and form the subjective value in the experiment who without knowledge of game. So the experimental economics have become one of the indispensable approaches. The classical game theory is to assume that the main body of the behavior is completely rational, that is, the subject can find the optimal strategy in the given condition and choose the best; In addition, it is assumed that the game rules are exogenous and given, and it is the common knowledge of the behavior subject, so the classical game theory is usually analyzed by logical reasoning. But the subjective game theory (Kaneko, $\mathrm{m}$, Kline, J) think that each behavior subject has the subjective cognition on the form of the game and their behaviors are in their subjective game model for the game. Behavior subjects can not only learn the rival's strategies distribution in the game of a given form, also can use the interactive strategy to learn and update subjective game rules. So researchers usually analyze the subjective game theory through economic experiments, as Camerer's behavior game theory, Crawford explained: the experimental data is the most important source of experimental information we have. Gerber usually analysis the participant's learning process of game structure through the experimental design, what's more, the participant is in the absence of feedback information. Feltovich through the experimental design concluded that the feedback of opponent's strategy and gain information are the most important factors for the participants to make choice. These experimental studies were all constructed the corresponding experimental environments, the participants were completely separated but remained in the same round, what's more, at the same time the same experiment environment the participants' information are completely consistent.

In order to satisfy the multiple games research, we designed the multiple games experiment platform. Participants took part in the multiple games experiment without communication. Participants were anonymous paired and only can observe themselves' computer terminals, all instructions and the feedback were transferred by computer terminal. Participants can learn the game structure and form their own subjective opinions only through the feedback of their choices and results. At present, some researchers have carried out empirical researches based on the subjective game. Grimm Veronika and Mengel Friederike, Stahl and Haruvy Van have constructed the 
corresponding game experimental platform to collect game experimental data. However, our research is based on the subjective game, through the design of the experimental platform realized multiple games, so as to observe the interaction of different game structures under the subjective game and the different results under the different match mechanisms. In order to achieve our vision, in this paper, the experimental platform of the administrator can change the number of game structure and match mechanism, finally we can collect all the experiment data analyze and compare behavior rules, draw relevant conclusions. Need to stress is that this experimental platform, the administrator change the game structure is by changing the number of the percentage of the game structure, and the game structure in experimental process is random.

\section{Platform Architecture}

The platform is combined with visual studio SQL database, using C\# language, designed and implemented of multiple games platform based WEB, which will be used to record the experimental data for our study of the subjective game theory.

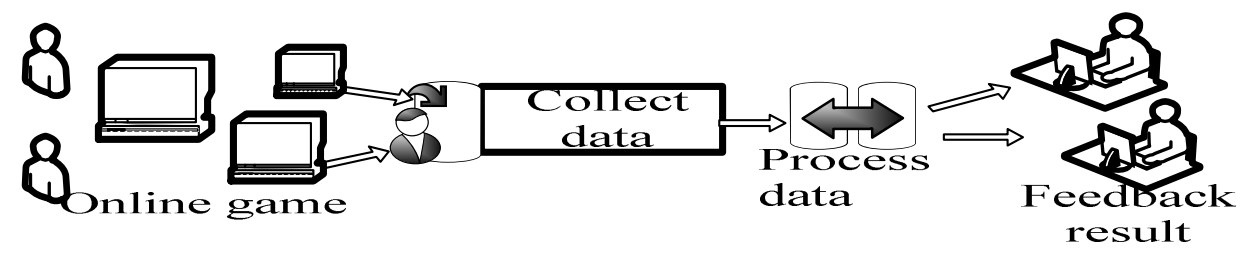

Fig.1, Platform architecture diagram

Participants Operation. First, the participants were distributed to the computer terminal, and read the experiment instructions. After the start of the experiment, the participants first register their accounts, then landing. After landing participants will face only two options on the selection page, they can select one of the options, the data will be submitted to the user database, and finally wait for the feedback results. Administrator refreshes the user database in the background, once submitted all, user data will be placed in an array of $2 \times 2$, the system will according to a given game structure to matching the $2 \times 2$ array, a set of matching results are given to the correspond user. Users can learn the game structure based on feedback data, feedback data page will redirect into the next round of selection page after 10 seconds, so again and again until to the end of the regulation on the number of rounds.

Administrator Operation. Administrator before the experiment need to set up the proportion of each game structure, in the corresponding random game structure to fill in the number of rounds, to realize the different game structures of multiple mixed. Such as $20 \%$ of the prisoner's dilemma game, $80 \%$ of the stag hunt game. That is a total of 50 rounds in random, the prisoner's dilemma game 10 rounds the stag hunt game 40 rounds. Administrators also need to set the game results match method, select the corresponding button, 50 rounds in matching of both the fixed pair and 50 rounds in matching of both the random pair. What's more, administrator would still need to enter the number of participants, which is used to testing whether the data are submitted.

\section{Function Module}

User Module. The user module has four sub modules: registration module, submit module, benefit module, feedback module.

Registration Module. Users register their own account first, then according to their own registered account to login.

Submit Module. After completion of the user login, enter the selection page, each page, there are two options: $\mathrm{X}$ and $\mathrm{Y}$, the user must select one of these options can click submit, then wait for feedback. 
Benefit Module. The waiting page will judge the database whether the database of two-dimensional array data is completed, the data match the completion the benefit of the page will automatically jump.

Feedback Module. Benefit feedback is according to the user's user name search database matching good calculation results, the feedback page will be displayed on the choice of the user himself on the choice page, user's own benefit and the opponent's benefit of the user matches.

Administrator Module. Administrator module has five modules, respectively is: set parameters module, the user table management module, select table data module, the matching data table module, the record data module.

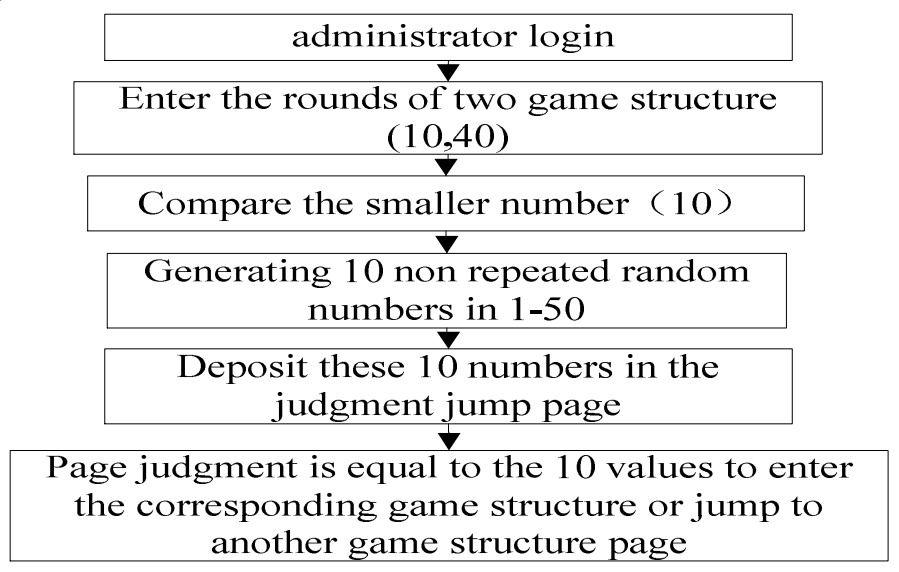

Fig.2, Flow chart of multiple games

Set Parameters Module. Before the start of the experiment, the administrator needs to set up the game structure of the proportion of the display, that is, the number of the corresponding game structure appears, select the matching method of the experimental data and the number of players involved in the game.

User Table Management Module. User table module shows the user's registration information, if there are problems or errors, the administrator can delete, modify the data.

Select Table Data Module. The select module shows the users' choice of information, if the users' selection are error, the administrator can modify in the background.

Matching Data Table Module. After each round of selection, the administrator according to the current game structure matching the choice of table data fixed or random, calculating the results table.

Record Data Module. For game experiment is repeated, so the users' select information and the benefit results table of each round needs to be clear after feedback, in order to convenient the next round of continue to store data calculation the record table module preserves all the data.

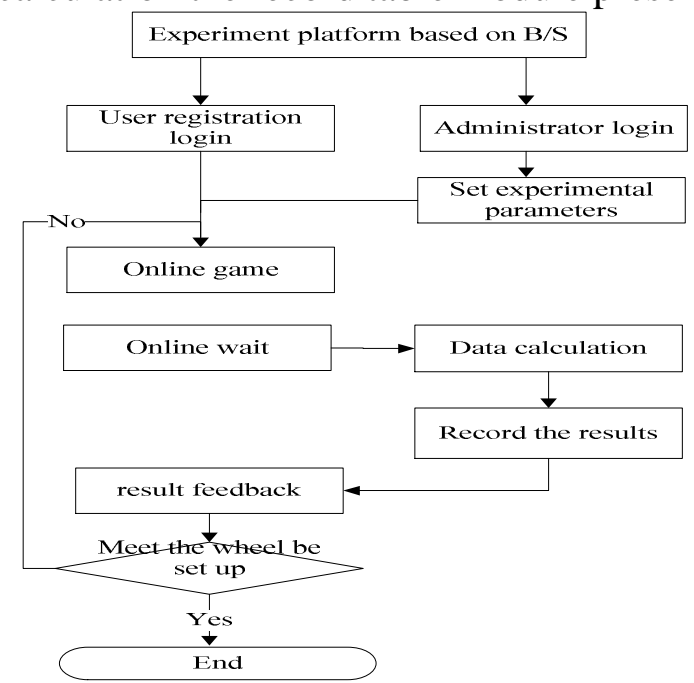

Fig.3, Flow chart of the experimental platform 


\section{Software Architecture}

Platform using the MVC framework MVC is refers to the model view and controller the three parts. It is a software development framework, the user interface layer, business logic layer and data layer separation so that it makes the code easier to maintain.

User Interface Layer. It is mainly deal with the operation of the game player. This layer is mainly synchronized with the background data, and provide interface for these data.

Business Logic Layer. It is mainly to realize the business logic of the main body of the game and the administrator, according to the different logic needs of the player and the administrator, so as to choose different methods to achieve.

Data Layer. The data layer is mainly operating the database, such as the display of the game data, update the game database, delete game data, etc.

\section{Database Design}

Database design is in a given application environment, establishing the most suitable and optimization database for the application system. It is to meet the needs of the game players and administrators of various applications (information requirements and processing requirements).

Data Sheet. Multiple games experiment platform is established by game users table, subject selection table, matching table, record table and parameter setting table.

Users Table. The table is storing users' information, user id, user name, the user password and user gender, they are not allowed to be empty.

Selection Table. The table is storing users' selection information of each round, including user number, user name, the user to select, round number four fields, to be sure that the choose table immediately emptying after putting in the matching table, which is used to store the next round of selection.

Matching Table. The data from selection table are stored in the form of a two-dimensional array, and calculated the matching results, including user number, user name, the user to select, round number and the results of five fields. Matching table empty immediately after the end of each round too, it is used to store the next round of selection.

Record Table. Record table with matching table fields is consistent, but the record store all round the number of data and the table does not have primary key.

Parameter Setting Table. The table is stored the rounds of different game structure which is inputted by the administrator. It is also storing the way of matching and the number of players involved in the game, before the start of each experiment to set, and then start the experiment.

ADO.NET Data Access Technology. ADO.NET code is strong, it can be maintained, its performance is good, what's more, it is a kind of powerful high performance data technology.

There are two basic components of ADO.NET object model: data sets and data providers. Data sets and game data source disconnected and does not require know the source of the saved game data; Net data provider and game data source connection, and execute SQL command for the game data sources. Access pattern is shown in Fig.4.

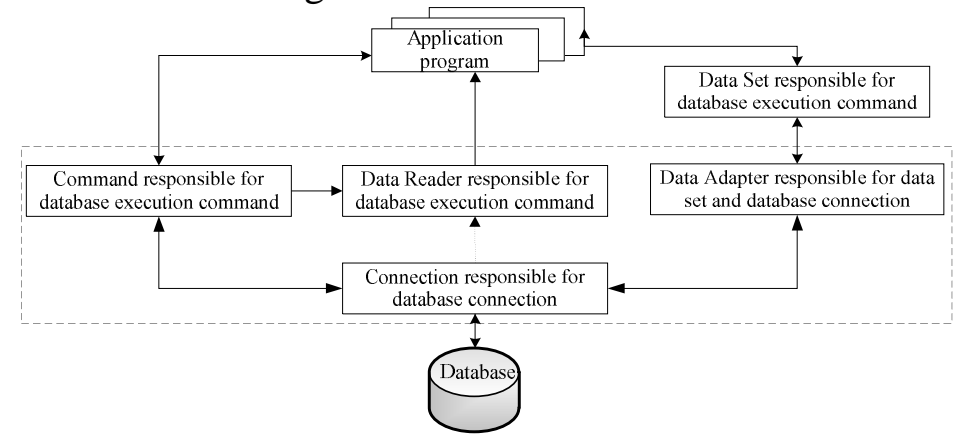

Fig.4, Data access patterns 


\section{Platform Implementation}

The CSS, HTML and other related technologies is applied to the interface design of the multiple games experiment platform. Platform is using B / S architecture and MVC development model, combining with HTML, CSS to achieve the static display for foreground and background game data, through ADO accessing database SQL, using JavaScript, Ajax and J Query technology for multiple games platform providing convenient game data exchange, achieving the subject to select and gain benefit, the administrator to calculate result data and dynamic feedback.

Platform two matching modes are essentially change the way to take out the data. When the fixed matching is selected, the table data is stored in the matching table, and the sequence is unchanged. But random matching, the data of the order of is random, so as to achieve a random match. Code as follows:

Select * form Submit1

Select * from Submit1 order by newid().

With the administrator interface game data record table as an example, the record table interface is through the view Grid control, the background data is displayed on the page through the corresponding field matching, and realized the background and the game database binding.

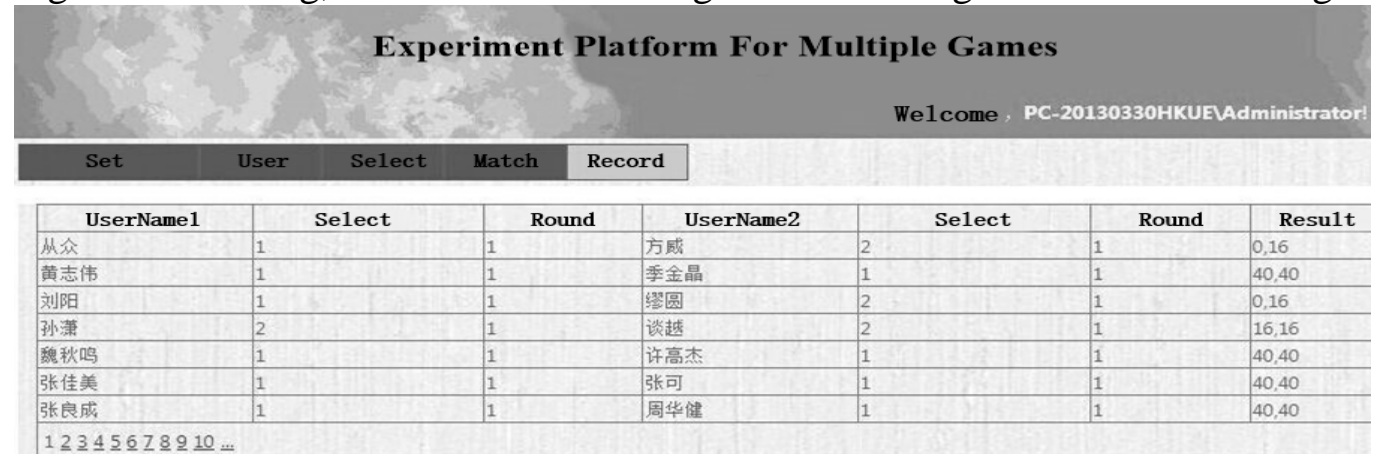

Fig.5, Administrator interface

\section{Summary}

This platform can collect the experimental data and calculate the feedback in time when the participants are completely separated. The administrator can realize the multiple games through parameter setting. The platform is combined with.NET, SQL database and B/S system architecture and other technologies, and these technologies are integrated into the WEB platform. These technologies are greatly improving the efficiency of our experiments, also ruling out a lot of interference factors. In addition our experimental design with the previous similar studies of experimental design is completely different, this platform has realized the two game structures in random mixed at the set proportion and switching matching random. This platform has fully realized in one experiment mixed multiple different game structures, it provides an experimental platform for our research. Such standardized data collection making our analysis results more reliable and can response the authenticity of the data. This way of the experiment is worth continuing to explore and improve.

\section{References}

[1]Colin F Camerer Behavioral Game Theory Experiments in Strategic Interaction[M].Zhongguo Renmin Daxue Chubanshe.(2011)

[2]E.Haruvy, D.O.Stahl, Between-game rule learning in dissimilar symmetric normal-form games[J], Games Econ.Behav. 2011,74 (1): 208-221.

[3]Feltovich,N. Effect of matching on learning in games played under limited information[J]. Pacific Economic Review. 2014 (19). 
[4]Grimm, Veronika and Friederike Mengel, "An experiment on learning in a multiple games environment[J]," Journal of Economic Theory, 2012, 147:2220-2259.

[5]Gerber,A.Learning in and about Games[J]. Working Paper. 2006.

[6]John Duffy, Dietmar Fehr,Equilibrium Selection in Similar Repeated Games:Experimental Evidence on the Role of Precedents[J],Working Paper.(2014)

[7]Kaneko,M., Kline,J. Inductive game theory: A basic scenario[J].Journal of Mathematical Economics.2008 (44).

[8]George Shepherd,Asp.Net Step by Step[M].Beijing:Qinghua Daxue Chubanshe.(2011) 